\title{
Comparative study of the influence of diabetes distress and depression on treatment adherence in Chinese patients with type 2 diabetes: a cross- sectional survey in the People's Republic of China
}

\author{
This article was published in the following Dove Press journal: \\ Neuropsychiatric Disease and Treatment \\ 30 August 2013 \\ Number of times this article has been viewed
}

\author{
Jie Zhang' \\ Cui-ping $X u^{2}$ \\ Hong-xia $\mathrm{Wu}^{2}$ \\ Xiu-juan $X u e^{2}$ \\ Ze-jun $\mathrm{Xu}^{3}$ \\ Yan $\mathrm{Li}^{4}$ \\ Qing $\mathrm{Gao}^{2}$ \\ Qing-zhi Liu ${ }^{5}$ \\ 'Shandong Medical College, \\ Jinan, People's Republic of China; \\ ${ }^{2}$ Qianfoshan Hospital, Shandong \\ University, Jinan, Shandong, People's \\ Republic of China; ${ }^{3}$ Sichuan Provincial \\ People's Hospital, Chengdu, Sichuan, \\ People's Republic of China; ${ }^{4}$ Shandong \\ University of Traditional Chinese \\ Medicine, Jinan, Shandong, People's \\ Republic of China; ${ }^{5} \mathrm{QiLu}$ Hospital, \\ Shandong University, Jinan, Shandong, \\ People's Republic of China
}

Correspondence: Cui-ping Xu Qianfoshan Hospital, Shandong University, Jinan, Shandong, People's Republic of China Email xucuiping775@sohu.com
Background: The purpose of this study was to explore diabetes-related distress and depression and their influence on treatment adherence in Chinese patients with type 2 diabetes.

Methods: We surveyed 200 type 2 diabetic patients from two public hospitals using the Diabetes Distress Scale (DDS), Zung Self-rating Depression Scale, and Revised Treatment Adherence in Diabetes Questionnaire (RADQ). A multiple regression model was used to explore the relationship between diabetes distress, depression, and treatment adherence.

Results: In the 200 eligible patients, the incidence of depression and diabetes distress was approximately $24 \%$ and $64 \%$, respectively. The mean score on the RADQ was $23.0 \pm 6.0$. Multiple regression analysis showed that DDS scores $(\beta=5.34, P=0.000)$, age $(\beta=0.15$, $P=0.014)$, and family history $(\beta=3.2, P=0.016)$ had a positive correlation with depression. DDS scores $(\beta=-2.30, P=0.000)$ and treatment methods $(\beta=-0.93, P=0.012)$ were risk factors for poor treatment adherence, whereas age $(\beta=0.089, P=0.000)$ and cohabitation $(\beta=0.93, P=0.012)$ increased treatment adherence. The independent-samples $t$-test showed that depression also affected treatment adherence $(t=2.53, P<0.05)$.

Conclusion: These findings suggest that the DDS is a predictor of depression and that diabetes distress plays a more important part than depression in treatment adherence. Screening for diabetes distress may be useful for primary prevention of psychologic problems; however, some form of specialized psychologic intervention should be incorporated to promote patient adherence with treatment.

Keywords: distress, depression, type 2 diabetes, treatment, adherence, People's Republic of China

\section{Introduction}

The association between mental health and type 2 diabetes is well known, and research has shown that the prevalence of depression in patients with diabetes is 1.5-3.0 times higher than in the general population. ${ }^{1,2}$ Depression has been shown to affect diabetes treatment outcomes and patient self-management behaviors. However, there is some evidence showing that the negative influence of depression on diabetes could also be explained by diabetes distress. ${ }^{3}$ Diabetes distress is defined as patient concern about disease management, support, emotional burden, and access to care, ${ }^{4}$ and has been considered as part of a more global approach to the psychologic issues associated with diabetes. ${ }^{5}$ Recently, comparative studies of diabetes distress and depression have been conducted. Fisher et al found that $70 \%$ of patients with diabetes who displayed high levels of depressive affect were not necessarily clinically depressed, ${ }^{6,7}$ but were 
experiencing high levels of emotional distress stemming from concerns and worries about their diabetes and its management. ${ }^{6,8}$ Further, Gonzalez et al surveyed 879 patients with type 2 diabetes and found that even low levels of depressive symptoms were associated with nonadherence to important aspects of diabetes self-care. ${ }^{9}$

Diabetes distress, depression, and subclinical depression are all psychologic disorders affecting patients with diabetes. A previous study reported that improvement in glycemic control following a diabetes education intervention was associated with a change in diabetes distress but not a change in depressive symptoms, ${ }^{10}$ and other research has shown that diabetes distress but not depression or depressive symptoms is associated with glycemic control. ${ }^{11}$ Diabetes distress is not necessarily a sign of depression, but is associated with a range of psychiatric disorders. However, most interventions concerning psychologic health in patients with diabetes are based on the depression literature, suggesting a need to consider different interventions for diabetes patients who are distressed but not clinically depressed, ${ }^{6}$ especially in the People's Republic of China, where research on diabetes distress is rare.

Treatment of diabetes is a major undertaking, and adherence with advice from health care providers is key to the success of treatment. Previous research has demonstrated the influence of diabetes distress on glucose control. ${ }^{12}$ However, we feel that treatment adherence not only incorporates glucose control, and we speculated only the glucose control could not reflect patient adherence comprehensively. Health education on diabetes has become an essential component of treatment for the disease, and psychologic nursing has been used to intervene in patients with depression, resulting in an obvious improvement in treatment adherence. We hypothesized that diabetes distress has a greater effect than depression on treatment adherence. In this study, we revised the adherence in diabetes questionnaire to reflect treatment of diabetes in the People's Republic of China, ie, diet, exercise, and self-monitoring (glucose control was included), as well as frequency of checks, other biochemistry tests, and dealing with diabetes-related emergencies in daily life. Using the Revised Adherence in Diabetes Questionnaire (RADQ), we investigated how diabetes distress and depression influenced patient adherence with treatment and compare their actions.

\section{Materials and methods Design and sample}

Following the requirements for a convenience sample, we selected the two public hospitals in Jinan, Shandong, the
People's Republic of China, which see the largest number of patients. Patients with type 2 diabetes who visited these two hospitals between December 2011 and April 2012 were invited to participate in this cross-sectional survey. Inclusion criteria were age 18-80 years, diagnosis of diabetes for at least 6 months, ability to communicate fluently and clearly, and without dementia, psychosis, or severe complications of diabetes (eg, on dialysis). Patients who met the inclusion criteria and provided their informed consent were recruited to complete the questionnaire. Two hundred of 225 eligible patients completed the questionnaire. Demographic and clinical characteristics canvassed in our questionnaire included age, sex, cohabitation, educational level, duration of diabetes, treatment regimen, and status of present glucose control.

\section{Survey instruments Chinese version of Diabetes Distress Scale}

The Chinese version of the Diabetes Distress Scale (CDDS) was developed by translating the original Diabetes Distress Scale ${ }^{13}$ into Chinese. ${ }^{14}$ The reliability and validity of the CDDS had been validated in the People's Republic of China, ${ }^{15}$ and our own research has shown this test to have good reliability, with a Cronbach's alpha of 0.903. The CDDS is a 17-item instrument representing the distress experienced over the previous month using a Likert scale, with each item scored from 1 (no distress) to 6 (serious distress) and comprising four subscales, ie, emotional burden (five items), physician-related distress (four items), regimen-related distress (five items), and interpersonal diabetes-related distress (three items). According to the revised rating system developed by Fisher et al, a mean item score $\leq 2$ indicates no distress, $2-3$ indicates moderate distress, and $>3$ indicates severe distress. ${ }^{16} \mathrm{~A}$ total scale can be calculated, with higher scores indicating greater distress. ${ }^{17}$

\section{Self-rating Depression Scale}

The Self-rating Depression Scale (SDS) ${ }^{18}$ has been confirmed to be a valid and reliable instrument in several studies, ${ }^{19,20}$ and in our research had shown good reliability, with a Cronbach's alpha of 0.796 . This assessment tool is a self-reported 20-item measurement of symptoms of depression. Item responses are ranked from 1 to 4 , with higher scores corresponding to more frequent symptoms for each item (1, rarely; 2 , some of the time; 3 , very often/often; 4 , almost always). The item scores are multiplied by 1.25 to obtain a standard 4-100 scale with only the integral section; the severity of depression is divided into four grades, with $0-52$ indicating 
"not depressed", 53-62 indicating "slightly depressed", 63-72 indicating "moderately depressed", and $\geq 73$ indicating "severely depressed".

\section{Revised Adherence in Diabetes Questionnaire}

We used the RADQ to assess patients' adherence with treatment, including diet, exercise, medicine, self-monitoring, and the frequency of reexaminations. The 10-item RADQ, modified by Chen et al, ${ }^{21}$ is a self-administered questionnaire. Item responses are ranked from 1 to 4 , with higher scores corresponding to better behaviors (1, rarely; 2, occasionally; 3 , often; 4, always). The questionnaire has a minimum score of 10 and a maximum score of 40, with higher scores indicating better adherence. Our research has demonstrated this test to have good reliability, with a Cronbach's alpha of 0.872 .

\section{Ethical approval}

The study was approved by the ethics committee at Qianfoshan Hospital (YLS2012/46). The patients and their relatives were informed verbally and in writing about the study protocol and could decline to participate if they wished. The data were coded and anonymized before statistical analysis.

\section{Statistical analysis}

We used descriptive statistics to analyze demographic covariates and CDDS, SDS, and RADQ scores. To adjust for confounding variables such as demographic factors, a multiple regression model was used to assess the effects of diabetes distress on depression and the influence of diabetes distress and depression on treatment adherence. The independentsamples $t$-test was used to assess for a difference in RADQ scores between depressed and nondepressed patients. The level of statistical significance was set at $P<0.05$. All statistical analysis was carried out using Statistical Package for the Social Sciences version 17.0 software (SPSS Inc, Chicago, IL, USA).

\section{Results}

The characteristics of the 200 patients (96 male, 104 female) with type 2 diabetes are shown in Table 1 . The age of the patients ranged from 22 to 80 years, and their mean duration of diabetes was 9 years. The majority (73\%) of the study population received both medical therapy and lifestyle intervention. Their scores on the SDS, CDDS, and RADQ are also shown in Table 1. Considering an SDS score of 53 to be diagnostic, the incidence of depression was approximately $24 \%$, and almost $64 \%$ of the patients experienced moderate
Table I Demographic and clinical characteristics of 200 patients and survey instrument results

\begin{tabular}{|c|c|}
\hline & Value \\
\hline \multicolumn{2}{|l|}{ Sex, n (\%) } \\
\hline Men & $96(48 \%)$ \\
\hline Women & $104(52 \%)$ \\
\hline Age, years mean (SD) & $59.6(11.0)$ \\
\hline Diabetes duration, mean (SD) & $9.0(7.7)$ \\
\hline \multicolumn{2}{|l|}{ Education level, n (\%) } \\
\hline Illiterate or primary school & $34(17 \%)$ \\
\hline Junior high school & 38 (19\%) \\
\hline Senior high school or university & $128(64 \%)$ \\
\hline \multicolumn{2}{|l|}{ Family history, n (\%) } \\
\hline Yes & $6 \mathrm{I}(30.5 \%)$ \\
\hline No & 139 (69.5\%) \\
\hline \multicolumn{2}{|l|}{ Complication, n (\%) } \\
\hline Yes & $92(46 \%)$ \\
\hline No & $108(54 \%)$ \\
\hline \multicolumn{2}{|l|}{ Treatment, n (\%) } \\
\hline Diet and exercise only & $13(6.5 \%)$ \\
\hline Medicine & $40(20 \%)$ \\
\hline Both the above & I 47 (73.5\%) \\
\hline \multicolumn{2}{|l|}{ Cohabitation, n (\%) } \\
\hline Single living & $12(6 \%)$ \\
\hline Living with a partner/other & $188(94 \%)$ \\
\hline \multicolumn{2}{|l|}{ Level of depressive symptoms } \\
\hline Slight, n (\%) & $152(76 \%)$ \\
\hline Mild, n (\%) & $34(17 \%)$ \\
\hline Moderate, n (\%) & II (5.5\%) \\
\hline Severe, n (\%) & $3(1.5 \%)$ \\
\hline \multicolumn{2}{|l|}{ Level of diabetes distress } \\
\hline Slight, n (\%) & $72(36 \%)$ \\
\hline Moderate, n (\%) & $93(46.5)$ \\
\hline Severe, n (\%) & $35(17.5 \%)$ \\
\hline RADQ score (SD) & $23(6.0)$ \\
\hline
\end{tabular}

Abbreviations: RADQ, Revised Treatment Adherence in Diabetes Questionnaire; $\mathrm{SD}$, standard deviation.

to severe distress in their daily life. The mean RADQ score was only 23 .

\section{Relationship between diabetes distress and depression}

We used the Pearson's correlation test to assess the relationship between depression and diabetes distress, and found a significant correlation $(r=0.318, P<0.01)$. A multiple regression model was used to explore the influence of diabetes distress on depression and to control for the effects of potential confounders such as demographic variables. The results shown in Table 2 indicate that higher family income and aging were associated with lower SDS scores $(\beta=-0.529, P<0.05$ and $\beta=-0.43$, respectively, $P=0.014)$. Factors that significantly increased depression were CDDS scores $(\beta=5.34, P=0.000)$ and family history $(\beta=3.2, P=0.016)$. 
Table 2 Relationship between depression and diabetes distress

\begin{tabular}{llll}
\hline & $\begin{array}{l}\text { Standardized } \\
\text { coefficients }\end{array}$ & $\mathbf{9 5 \%} \mathbf{C l}$ & P-value \\
\hline DD & 5.34 & $3.53-7.15$ & 0.000 \\
Age & -0.43 & -0.61 to -0.25 & 0.014 \\
Family history & 3.2 & $0.6-5.8$ & 0.016 \\
Family income & -0.529 & -1.05 to -0.003 & 0.049 \\
\hline
\end{tabular}

Abbreviations: $\mathrm{Cl}$, confidence interval; $\mathrm{DD}$, diabetes distress.

\section{Influence of diabetes distress and depression on treatment adherence}

We used a multiple regression model to determine whether diabetes distress contributed adversely to adherence with treatment for diabetes, in which the RADQ score was considered as a dependent variable, while the demographic variables, CDDS scores, and the SDS scores were considered to be independent variables. As shown in Table 3, higher levels of diabetes distress and more complex treatment methods indicated poorer treatment adherence $(\beta=-2.30, P=0.000$ and $\beta=-0.93, P=0.012$, respectively), whereas aging and cohabitation increased treatment adherence $(\beta=0.089, P=0.000$ and $\beta=0.93, P=0.012$ ). However, depression was deleted from the model.

\section{Difference of depressed and nondepressed in RADQ score}

Table 4 shows that both diabetes distress and depression were related to RADQ scores. Table 3 indicates that depression did not contribute to adherence with treatment for diabetes. However, diabetes distress was shown to increase the risk of depression and to reduce treatment adherence. Therefore, we used the independent-samples $t$-test to assess whether depression had an adverse effect on treatment adherence. As shown in Table 4, there were differences in RADQ scores between depressed and nondepressed patients $(t=2.53, P<0.05)$.

\section{Discussion}

Depression and diabetes distress are used widely as indicators of psychologic state in patients with type 2 diabetes. Some studies have demonstrated the influence of diabetes distress on diabetes, ${ }^{12}$ but such research is rare in the People's Republic

Table 3 Diabetes distress and treatment adherence of diabetes

\begin{tabular}{llll}
\hline & $\begin{array}{l}\text { Standardized } \\
\text { coefficients }\end{array}$ & $\mathbf{9 5 \%} \mathbf{C l}$ & P-value \\
\hline DD & -2.30 & -3.40 to -1.21 & 0.000 \\
Age & 0.089 & $0.013-0.164$ & 0.021 \\
Cohabitation & 0.994 & $0.023-1.836$ & 0.044 \\
Treatment & -0.93 & -1.77 to -0.22 & 0.012 \\
\hline
\end{tabular}

Abbreviations: $\mathrm{Cl}$, confidence interval; $\mathrm{DD}$, diabetes distress. of China. We first investigated the present status of diabetes distress and depression simultaneously, and found that there were significant differences between the two. In our research, the prevalence of depression was about $24 \%$, which is similar to that reported by Odusan et al. ${ }^{22}$ However, using the revised rating proposed by Fisher et al, ie, " 2 score was the cut-off point", ${ }^{16}$ the prevalence of diabetes distress was almost $64 \%$, indicating that disease management and self-care are emotionally burdensome for the majority of diabetes patients and that diabetes distress is more prevalent than depression in these patients. This was the first comparative study of the prevalence of diabetes distress and depression in type 2 diabetics. Further, although low family income and aging were risk factors for depression in our study, there is a suggestion that diabetes distress is another risk factor, which is similar to the observation made by Pouwer et al that "the occurrence of depression in diabetes patients was related to diabetes distress", ${ }^{23}$ so, only assessing depression in diabetes patients, some patients with diabetes distress but not depression will be neglected. Meanwhile, our research perhaps explains the common phenomenon "why so many interventions based on depression have been conducted in diabetes patients, but the mental health was still the barrier to better treatment."

The influence of depression on adherence with dietary recommendations and medication for diabetes has been reported extensively. ${ }^{24,25}$ However, there was a striking difference in our investigation in that after adjustment for diabetes distress, depression was deleted from the multiple regression model, whereas diabetes distress was strongly correlated with treatment adherence (see Table 3). To our knowledge, this is the first report suggesting diabetes distress is a risk factor for poor treatment adherence, and is also the first to demonstrate that depression has little impact on treatment adherence in the People's Republic of China. We believe that this finding highlights a difference between diabetes distress and depression, and suggests that health educators need to focus on interventions based on diabetes distress.

Another notable feature of our study is its use of the RADQ which has been revised to be relevant for the Chinese health care system. Previous studies of adherence have been focused on diet, sport, medication, and glucose monitoring. However, the RADQ incorporates further biochemistry tests, frequency of reexaminations, and diabetes-related emergencies in daily life. Given that treatment adherence was a better reflection than glucose control to doctors' advice and nurses' health education, the proof that diabetes distress played a more important part in glucose control than depression, could not indicate that diabetes distress was also more important 
Table 4 Difference in RADQ score between depressed and nondepressed patients

\begin{tabular}{|c|c|c|c|c|}
\hline & \multicolumn{2}{|l|}{ Correlations/P } & \multirow[t]{2}{*}{ Depressed } & \multirow[t]{2}{*}{ Nondepressed } \\
\hline & Diabetes distress & Depression & & \\
\hline RADQ scores & $-0.423 / 0.000$ & $-0.285 / 0.009$ & $24.82 \pm 6.08$ & $25.06 \pm 5.40$ \\
\hline Diabetes distress & & $0.318 / 0.000$ & & \\
\hline$t$ & & & & \\
\hline$P$ & & & & \\
\hline
\end{tabular}

Abbreviation: RADQ, Revised Treatment Adherence in Diabetes Questionnaire.

in treatment adherence than depression. The present study underscores for caregivers the importance of diabetes distress in treatment adherence. In our investigation, the mean RADQ score was 23 , indicating a moderate level of distress, which is a confusing problem for caregivers in the People's Republic of China, given that it does not correspond with what the educators have contributed to the patients' education. However, we speculate that this apparent contradiction could be explained as follows: our diabetes education is inadequate; psychological state is key to treatment adherence, but not only or not all depression was our main intervening criteria, so our previous depression-oriented intervention was inadequate; and perhaps, as the report suggested, diabetes distress was more important than depression to treatment adherence, but was previously neglected.

Although our results for depression and adherence are in contrast with those reported by others, we do not disagree with earlier findings. We used the independent-samples $t$-test to test for differences in treatment adherence between depressed and nondepressed patients, and the result was contrary to what Table 3 shows. However, this apparent contradiction could be interpreted as an indicator that both diabetes distress and depression stem from diabetes self-care and are emotionally burdensome, which were some overlapping experiences, the previous findings about depression on adherence might be explained by diabetes distress, which was similar to Kokoszka et al. ${ }^{26}$ We conclude that diabetes distress plays a more important part in treatment adherence, because depression was deleted from the multiple regression model. However, we speculate that this explanation applies only to patients with minor depression, as for the major ones especially the patients with antidepressant, the depressed state will influence patients' treatment adherence seriously, simultaneously, another reason perhaps was a limitation of our investigation: the subjects in our study were only inpatients who did not have a diagnosis of depression in their medical records.

The results of this comparative study confirm our hypothesis that even low levels of depression can have a significant negative impact on treatment adherence and suggest that diabetes distress is an effective indicator of minor depression which could impair treatment adherence and self-management. Therefore, we suggest that some specialized psychological intervention, eg, using a diabetes distress scale and treating depression, would be an effective tool to prevent psychological problems and promote patient adherence with treatment.

\section{Conclusion}

This study documents the use of the CDDS in Chinese patients and encourages health care providers to undertake further international research on emotional distress in patients with diabetes. We compared the influence of diabetes distress and depression on patient adherence with treatment. Our results indicate that although diabetes-related distress may play a more important part than depression in patient adherence, both are risk factors for poor adherence. The findings of this study suggest that health care providers should differentiate between diabetes distress and depression, and that a primary health care program addressing psychological health in diabetes patients should be widely implemented.

\section{Acknowledgments}

This study was supported by a grant from Qianfoshan Hospital, Shandong Province. The authors thank the endocrinology nurses for their help with data collection and Professor Ju Liu for assistance with preparation of the manuscript.

\section{Author contributions}

Cui-ping $\mathrm{Xu}$, Jie Zhang, Hong-xia $\mathrm{Wu}$, and Xiu-juan Xue contributed to study design. Ze-jun Xu, Yan Li, Qing-zhi Liu and Qing Gao contributed to the acquisition of the data. Jie Zhang took charge of drafting the article. All authors were involved in the critical revising of the manuscript and final approval of the proof published.

\section{Disclosure}

The authors report no conflicts of interest in this work. 


\section{References}

1. Gavard JA, Lustman PJ, Clouse RE. Prevalence of depression in adults with diabetes. An epidemiological evaluation. Diabetes Care. 1993;16:1167-1178.

2. Nichols GA, Brown JB. Unadjusted and adjusted prevalence of diagnosed depression in type 2 diabetes. Diabetes Care. 2003;26:744-749.

3. Hermanns N, Kulzer B, Krichbaum M, Krichbaum M, Kubiak T, Haak T. How to screen for depression and emotional problems in patients with diabetes: comparison of screening characteristics of depression questionnaires, measurement of diabetes-specific emotional problems and standard clinical assessment. Diabetologia. 2006;49:469-477.

4. Fisher L, Glasgow RE, Mullan JT, Skaff MM, Polonsky WH. Development of a brief diabetes distress screening instrument. Ann Fam Med. 2008;6:246-252.

5. Egede LE, Dismuke CE. Serious psychological distress and diabetes: a review of the literature. Curr Psychiatry Rep. 2012;14:15-22.

6. Fisher L, Skaff MM, Mullan JT, et al. Clinical depression versus distress among patients with type 2 diabetes: not just a question of semantics. Diabetes Care. 2007;30:542-548.

7. Grigsby AB, Anderson RJ, Freedland KE, Clouse RE, Lustman PJ. Presence of anxiety in adults with diabetes: a systematic review. J Psychosom Res. 2002;53:1053-1060.

8. Fisher L, Skaff MM, Mullan JT, Arean P, Glasgow R, Masharani U. A longitudinal study of affective and anxiety disorders, depressive affect and diabetes distress among adults with type 2 diabetes. Diabet Med. 2008;25:1096-1101.

9. Gonzalez JS, Safren SA, Cagliero E, et al. Depression, self-care, and medication adherence in type 2 diabetes: relationships across the full range of symptom severity. Diabetes Care. 2007;30:2222-2227.

10. Zagarins SE, Allen NA, Garb JL, Welch G. Improvement in glycemic control following a diabetes education intervention is associated with change in diabetes distress but not change in depressive symptoms. J Behav Med. 2012;35:299-304.

11. Fisher L, Mullan JT, Arean P, Glasgow RE, Hessler D, Masharani U. Diabetes distress but not clinical depression or depressive symptoms is associated with glycemic control in both cross-sectional and longitudinal analyses. Diabetes Care. 2010;33:23-28.

12. Fisher L, Glasgow R, Strycker L. The relationship between diabetes distress and clinical depression with glycemic control among patients with type 2 diabetes. Diabetes Care. 2010;33:1034-1036.

13. Polonsky WH, Fisher L, Earles J, et al. Assessing psychosocial distress in diabetes: development of the diabetes scale. Diabetes Care. 2005;28: $626-631$.
14. Ting RZ, Nan H, Yu MW, et al. Diabetes-related distress and physical and psychological health in Chinese type 2 diabetic patients. Diabetes Care. 2011;34:1094-1096.

15. Yang Q, Liu XQ. Reliability and validity of the diabetes distress scale. Journal of Nursing (China). 2010;17:8-10. Chinese.

16. Fisher L, Hessler DM, Polonsky WH, Mullan J. When is diabetes distress clinically meaningful?: establishing cut points for the Diabetes Distress Scale. Diabetes Care. 2012;35:259-264.

17. Graue M, Haugstvedt A, Wentzel-Larsen T, Iversen MM, Karlsen B, Rokne B. Diabetes-related emotional distress in adults: reliability and validity of the Norwegian versions of the Problem Area in Diabetes Scale (PAID) and the Diabetes Distress Scale (DDS). Int J Nurs Stud. 2012;49:174-182.

18. Zung W, Durham NC. A self-rating depression scale. Arch Gen Psychiatry. 1965;12:63-70.

19. Barefoot JC, Brummett BH, Helms MJ, Mark DB, Siegler IC, Williams RB. Depressive symptoms and survival of patients with coronary artery disease. Psychosom Med. 2000;62:790-795.

20. Passik SD, Lundberg JC, Rosenfeld B, et al. Factor analysis of the Zung Self-Rating Depression Scale in a large ambulatory oncology sample. Psychosomatics. 2000;41:121-127.

21. Chen ZH, Zhao XD, Xu YS, Zhu CZ, Zhang HY, Ji MY. Observing compliance of patients with diabetes mellitus from two methods. Academic Journal of Kunming Medical College. 2000;4:67-69. Chinese.

22. Odusan O, Oqunsemi O, Olatawura MO. Common mental disorders among subjects with T2DM in Sagamu, Nigeria. Afr J Med Med Sci. 2012;41:141-145

23. Pouwer F, Skinner TC, Pibernik-Okanovic M, et al. Serious diabetesspecific emotional problems and depression in a Croatian-Dutch-English survey from the European Depression in Diabetes [EDID] research consortium. Diabetes Res Clin Pract. 2005;70:166-173.

24. Ciechanowski PS, Katon WJ, Russo JE. Impact of depression symptoms on adherence, function, and costs. Arch Intern Med. 2000;160: 3278-3285.

25. Kalserkar ID, Madhaven SS, Amonkar MM, et al. Depression in patients with type 2 diabetes: impact on adherence to oral hypoglycemic agents. Ann Pharmacother. 2006;40:605-611.

26. Kokoszka A, Pouwer F, Jodko A, et al. Serious diabetes-specific emotional problems in patients with type 2 diabetes who have different levels of comorbid depression: a Polish study from the European depression in diabetes (EDID) research consortium. Eur Psychiatry. 2009;24:425-430.
Neuropsychiatric Disease and Treatment

\section{Publish your work in this journal}

Neuropsychiatric Disease and Treatment is an international, peerreviewed journal of clinical therapeutics and pharmacology focusing on concise rapid reporting of clinical or pre-clinical studies on a range of neuropsychiatric and neurological disorders. This journal is indexed on PubMed Central, the 'PsycINFO' database and CAS.

\section{Dovepress}

The manuscript management system is completely online and includes a very quick and fair peer-review system, which is all easy to use. Visit http://www.dovepress.com/testimonials.php to read real quotes from published authors. 